\title{
Complex projective 3-fold with non-negative canonical Euler-Poincare characteristic
}

\author{
Meng Chen and Kang Zuo
}

Let $V$ be a complex non-singular projective 3-fold of general type with $\chi\left(\omega_{V}\right) \geq 0$ (resp. $\left.>0\right)$. We prove that the $m$-canonical map $\Phi_{\left|m K_{V}\right|}$ is birational onto its image for all $m \geq 14$ (resp. $\geq 8$ ). Known examples show that the lower bound $r_{3}=14($ resp. $=8)$ is optimal.

\section{Introduction}

We work over the complex number field $\mathbb{C}$.

In this paper, we study multi-canonical systems $\left|m K_{V}\right|$ on complex projective 3 -folds $V$ of general type.

On a smooth complex complete curve $C$ of genus $g(C) \geq 2$, it is well known that the 3-canonical map $\varphi_{3}:=\Phi_{\left|3 K_{C}\right|}$ is always an embedding. On a smooth minimal projective surface $S$ of general type, Bombieri [2] proved that the $m$-canonical map is a birational morphism for all $m \geq 5$. The work of Tsuji [31], Hacon-McKernan [13] and Takayama [28] says that there exists a universal constant $r_{3}$ such that the $r_{3}$-canonical map $\Phi_{r_{3}}$ is birational for all smooth projective 3-folds of general type. We note that Tsuji [31] announced a very large $r_{3}$. Recently Chen and the first author [5] have given an explicit $r_{3}(\leq 77)$.

Under extra assumptions there have been already the following optimal results about $r_{3}$ for minimal projective 3 -folds $X$ of general type with $\mathbb{Q}$-factorial terminal singularities:

- $\varphi_{5}:=\Phi_{\left|5 K_{X}\right|}$ is birational if either $X$ is Gorenstein (by Chen, Chen and Zhang [4]) or $p_{g}(X) \geq 4$ (by Chen [8]) or $K_{X}^{3} \gg 0$ (by Todorov $[30]) . r_{3}=5$ is optimal.

- $\varphi_{8}:=\Phi_{\left|8 K_{X}\right|}$ is birational if $p_{g}(X) \geq 2$ (by Chen [8]). $r_{3}=8$ is optimal.

- $\varphi_{7}$ is birational if $q(X)>0$ (by Chen and Hacon [6]). 
- $\varphi_{5}$ is birational if $\chi\left(\omega_{X}\right) \geq 0$ and $q(X)>0$ (by Chen and Hacon [7]). $r_{3}=5$ is optimal.

Going on the study of 3 -folds with $\chi(\omega) \geq 0$, we prove the following.

Theorem 1.1. Let $V$ be a non-singular projective 3-fold of general type with $\chi\left(\omega_{V}\right):=\sum_{i=0}^{3}(-1)^{i} h^{i}\left(V, \omega_{V}\right) \geq 0$, where $\omega_{V}$ is the canonical line bundle of $V$. Then the $m$-canonical map $\varphi_{m}$ is birational onto its image for all $m \geq 14$.

The following example of Iano-Fletcher shows that $r_{3}=14$ in Theorem 1.1 is optimal.

Example 1.2. (See [10, No. 19, p. 151]) The canonical hypersurface $X_{28} \subset \mathbb{P}(1,3,4,5,14)$ has three terminal quotient singularities, $p_{g}(X)=1$, $q(X)=h^{2}\left(\mathcal{O}_{X}\right)=0$ and $\chi\left(\omega_{X}\right)=-\chi\left(\mathcal{O}_{X}\right)=0$. A smooth model $V$ of $X_{28}$ has the invariant: $\chi\left(\omega_{V}\right)=0$ and $V$ is of general type. It is clear that $\varphi_{m}$ is birational for all $m \geq 14$ and that $\varphi_{13}$ is not birational. So the lower bound $r_{3}=14$ in Theorem 1.1 is sharp.

Our method has a direct consequence.

Corollary 1.3. Let $V$ be a smooth projective 3-fold of general type with $\chi\left(\omega_{V}\right)>0$. Then the m-canonical map $\Phi_{\left|m K_{V}\right|}$ is birational onto its image for all $m \geq 8$.

Example 1.4. (See [10, No. 12, p. 151]) Fletcher has a 3 -fold $X_{16} \subset$ $\mathbb{P}(1,1,2,3,8)$ on which the 8 -canonical map is birational and the 7-canonical map is not birational. A smooth model $V$ of $X_{16}$ has the invariant $\chi(\omega)=1$ and $V$ is of general type. $V$ has $r_{3}(V)=8$. Thus the lower bound $r_{3}=8$ in Corollary 1.3 is again optimal.

Note that 3-folds of general type with $\chi(\omega) \geq 0$ form an interesting class, around which there have been already some established works:

- Gorenstein minimal 3-folds of general type have $\chi(\omega)>0$;

- Any 3-fold of general type admitting a generically finite cover over an Abelian variety has $\chi(\omega)>0$ (see Green-Lazarsfeld [12]); 
- Any 3-fold of general type, admitting a representation $\rho: \pi_{1}(X) \rightarrow \mathrm{GL}$ such that the Shafarevich map $\operatorname{Sh}_{\rho}$ is generically finite, has $\chi(\omega) \geq 0$ (see Jost-Zuo [16]);

- Any 3-fold of general type with generically large fundamental group is conjectured to have $\chi(\omega)>0$ (see Kollár [20, Conjecture 18.12.1]).

It is clear that the key point in studying pluricanonical maps is to compute $P_{m}$ for $m>1$. One may do this on a minimal model according to Reid [23]. When $\chi(\omega)=-\chi(\mathcal{O}) \geq 0$, it is clear from Reid's formula that $P_{2}>0$. This makes it possible for us to prove effective birationality of $\varphi_{m}$. But to prove the optimal bound $r_{3}=14$ need more evolved argument, which is exactly what we have done in this paper. The case $\chi(\omega)<0$ has been treated with quite a different approach very recently by Chen and the first author [5].

\section{Notations and set up}

Since both the birationality of pluricanonical maps and $\chi(\omega)$ are birationally invariant, we may study a minimal model. By the 3-dimensional Minimal Model Program (MMP) (see [19,22], for instance), we only have to consider a minimal 3-fold $X$ of general type with $\mathbb{Q}$-factorial terminal singularities. Denote the Cartier index of $X$ by $r:=r(X)$ which is the minimal positive integer with $r K_{X}$ a Cartier divisor, where $K_{X}$ is a canonical divisor on $X$. The symbol $\equiv$ stands for the numerical equivalence of divisors, whereas $\sim$ denotes the linear equivalence and $=_{\mathbb{Q}}$ denotes the $\mathbb{Q}$-linear equivalence.

\subsection{Set up for $\left|m_{0} K_{X}\right|$.}

Assume $P_{m_{0}}(X):=h^{0}\left(X, \mathcal{O}_{X}\left(m_{0} K_{X}\right)\right) \geq 2$ for some positive integer $m_{0}>0$. We study the $m_{0}$-canonical map $\varphi_{m_{0}}$ which is a rational map.

First we fix an effective Weil divisor $K_{m_{0}} \sim m_{0} K_{X}$. Take successive blow-ups $\pi: X^{\prime} \rightarrow X$ (along non-singular centers), which exists by Hironaka's big theorem, such that

(i) $X^{\prime}$ is smooth;

(ii) the movable part of $\left|m_{0} K_{X^{\prime}}\right|$ is base point free;

(iii) the support of $\pi^{*}\left(K_{m_{0}}\right)$ is of simple normal crossings. 
Denote by $g_{m_{0}}$ the composition $\varphi_{m_{0}} \circ \pi$. So $g_{m_{0}}: X^{\prime} \longrightarrow W^{\prime} \subseteq \mathbb{P}^{P_{m_{0}}(X)-1}$ is a morphism. Let $X^{\prime} \stackrel{f_{m_{0}}}{\longrightarrow} B \stackrel{s}{\longrightarrow} W^{\prime}$ be the Stein factorization of $g_{m_{0}}$. We have the following commutative diagram.

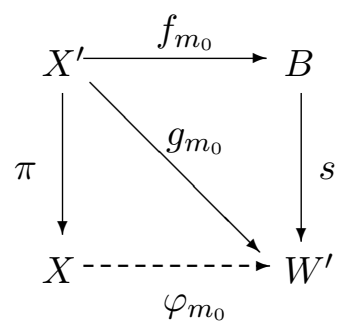

We recall the definition of $\pi^{*}\left(K_{X}\right)$. One has $r(X) K_{X^{\prime}}=\pi^{*}\left(r(X) K_{X}\right)+$ $E_{\pi}$, where $E_{\pi}$ is a sum of effective exceptional divisors. One defines $\pi^{*}\left(K_{X}\right):=K_{X^{\prime}}-(1 / r(X)) E_{\pi}$. So, whenever we take the round up of $m \pi^{*}\left(K_{X}\right)$, we always have $\left\ulcorner m \pi^{*}\left(K_{X}\right)\right\urcorner \leq m K_{X^{\prime}}$ for any integer $m>0$. We may write $m_{0} \pi^{*}\left(K_{X}\right)={ }_{\mathbb{Q}} M_{m_{0}}+E_{m_{0}}^{\prime}$, where $E_{m_{0}}^{\prime}$ is an effective $\mathbb{Q}$-divisor and $M_{m_{0}}$ is the movable part of $\left|m_{0} K_{X^{\prime}}\right|$. On the other hand, one has $m_{0} K_{X^{\prime}}={ }_{\mathbb{Q}} \pi^{*}\left(m_{0} K_{X}\right)+E_{\pi, m_{0}}=M_{m_{0}}+Z_{m_{0}}$, where $Z_{m_{0}}$ is the fixed part and $E_{\pi, m_{0}}$ an effective $\mathbb{Q}$-divisor which is a $\mathbb{Q}$-sum of distinct exceptional divisors. Clearly $Z_{m_{0}}=E_{m_{0}}^{\prime}+E_{\pi, m_{0}}$.

If $\operatorname{dim} \varphi_{m_{0}}(X)=2$, a general fiber of $f_{m_{0}}$ is a smooth projective curve of genus at least 2 . We say that $X$ is $m_{0}$-canonically fibered by curves.

If $\operatorname{dim} \varphi_{m_{0}}(X)=1$, a general fiber $S$ of $f_{m_{0}}$ is a smooth projective surface of general type. We say that $X$ is $m_{0}$-canonically fibered by surfaces with invariants $\left(c_{1}^{2}\left(S_{0}\right), p_{g}(S)\right)$, where $S_{0}$ is the minimal model of $S$. We may write $M_{m_{0}} \equiv a_{m_{0}} S$, where $a_{m_{0}} \geq P_{m_{0}}(X)-1$ by considering the degree of a curve in a projective space.

A generic irreducible element $S$ of $\left|M_{m_{0}}\right|$ means either a general member of $\left|M_{m_{0}}\right|$, whenever $\operatorname{dim} \varphi_{m_{0}}(X) \geq 2$ or, otherwise, a general fiber of $f_{m_{0}}$.

Definition 2.1. Assume that $\left|M^{\prime}\right|$ is movable on $V$. By abuse of concepts, we also define a generic irreducible element $S^{\prime}$ of an arbitrary linear system $\left|M^{\prime}\right|$ on an arbitrary variety $V$ in a similar way. A generic irreducible element $S^{\prime}$ of $\left|M^{\prime}\right|$ is defined to be a generic irreducible component in a general member of $\left|M^{\prime}\right|$.

\section{A technical theorem}

Believing that Theorem 2.2 in [8] is quite effective in treating 3-folds $X$ with $p_{g}(X) \geq 2$, we extend the technique there to build a parallel theorem so as 
to study those $X$ with $p_{g}(X) \leq 1$, but with $P_{m_{0}}(X) \geq 2$ for some integer $m_{0}>0$.

\subsection{Assumptions}

We need to make the following assumptions to explain our key method. Keep the same notation as in Section 2.1 above. Let $m>0$ be certain integer.

(i) Either $m \geq m_{0}+2$ and $p_{g}(X)>0$ or $\left|m K_{X^{\prime}}\right|$ separates different irreducible elements $S$ of $\left|M_{m_{0}}\right|$ (namely, $\Phi_{\left|m K_{X^{\prime}}\right|}\left(S^{\prime}\right) \neq \Phi_{\left|m K_{X^{\prime}}\right|}\left(S^{\prime \prime}\right)$ for two different irreducible elements $S^{\prime}, S^{\prime \prime}$ of $\left.\left|M_{m_{0}}\right|\right)$ and $p_{g}(X)=0$.

(ii) Assume that, on the smooth surface $S$, there is a movable linear system $|G|$ and that $C$, as a generic irreducible element of $|G|$, is smooth. The linear system $\mid m K_{X^{\prime}} \|_{S}$ on $S$ (as a sub-linear system of $\left|m K_{X^{\prime}}\right|_{S} \mid$ ) separates different generic irreducible elements of $|G|$. Or sufficiently, the complete linear system

$$
\left|K_{S}+\left\ulcorner(m-1) \pi^{*}\left(K_{X}\right)-S-\frac{1}{p} E_{m_{0}}^{\prime}\right\urcorner\right|_{S} \mid
$$

separates different generic irreducible elements of $|G|$.

(iii) There is a rational number $\beta>0$ such that $\left.\pi^{*}\left(K_{X}\right)\right|_{S}-\beta C$ is numerically equivalent to an effective $\mathbb{Q}$-divisor; Set

$$
\alpha:=\left(m-1-\frac{m_{0}}{p}-\frac{1}{\beta}\right) \xi
$$

and $\alpha_{0}:=\ulcorner\alpha\urcorner$.

(iv) Either the inequality $\alpha>1$ holds; or $C$ is non-hyperelliptic, $m-1-$ $\left(m_{0} / p\right)-(1 / \beta)>0$ and $C$ is an even divisor on $S$.

(v) Either $\alpha>2$; or $\alpha_{0} \geq 2$ and $C$ is non-hyperelliptic; or $C$ is nonhyperelliptic, $m-1-\left(m_{0} / p\right)-(1 / \beta)>0$ and $C$ is an even divisor on $S$.

Set $\xi:=\left(\pi^{*}\left(K_{X}\right) \cdot C\right)_{X^{\prime}}$ which is a positive rational number and define

$$
p:= \begin{cases}1 & \text { if } \operatorname{dim}(B) \geq 2, \\ a_{m_{0}}(\text { see Section } 2.1 \text { for the definition) } & \text { otherwise }\end{cases}
$$

Let $f:=f_{m_{0}}: X^{\prime} \longrightarrow B$ be an induced fibration by $\varphi_{m_{0}}$. 
Theorem 3.1. Let $X$ be a minimal projective 3-fold of general type with $P_{m_{0}}(X) \geq 2$ for some integer $m_{0}>0$. Keep the same notation as in Section 2.1 above. Then the inequality

$$
m \xi \geq 2 g(C)-2+\alpha_{0}
$$

holds under assumptions (iii) and (iv). Furthermore, $\varphi_{m}$ of $X$ is birational onto its image under assumptions (i), (ii), (iii) and (v).

Proof. First we shall show that $\left|m K_{X^{\prime}}\right|$ can separate different irreducible elements of $\left|M_{m_{0}}\right|$. When $p_{g}(X)=0,\left|m K_{X^{\prime}}\right|$ can separate different irreducible elements of $\left|M_{m_{0}}\right|$ by assumption (i). When $p_{g}(X)>0$, we consider the sub-system

$$
\left|K_{X^{\prime}}+\left\ulcorner(m-1) \pi^{*}\left(K_{X}\right)-\frac{1}{p} E_{m_{0}}^{\prime}\right\urcorner\right| \subset\left|m K_{X^{\prime}}\right| .
$$

Let $S^{\prime}$ and $S^{\prime \prime}$ be two different generic irreducible elements of $\left|M_{m_{0}}\right|$. Clearly one has

$$
K_{X^{\prime}}+\left\ulcorner(m-1) \pi^{*}\left(K_{X}\right)-\frac{1}{p} E_{m_{0}}^{\prime}\right\urcorner \geq K_{X^{\prime}}+\left\ulcorner\left(m-m_{0}-1\right) \pi^{*}\left(K_{X}\right)\right\urcorner+S \geq S
$$

by assumption (i). So $\left|K_{X^{\prime}}+\left\ulcorner(m-1) \pi^{*}\left(K_{X}\right)-\frac{1}{p} E_{m_{0}}^{\prime}\right\urcorner\right|$ can separate $S^{\prime}$ and $S^{\prime \prime}$ if either $\operatorname{dim}(B) \geq 2($ cf. $[29$, Lemma 2]) or $\operatorname{dim}(B)=1$ and $g(B)=0$ $((\mathrm{P} 2)$ in $[9$, Section 2.1]). For the case $\operatorname{dim}(B)=1$ and $g(B)>0$, one has $a_{m_{0}} \geq P_{m_{0}} \geq 2$. Thus $p \geq 2$. Since

$$
m-1-\frac{2 m_{0}}{p} \geq 1+\left(1-\frac{2}{p}\right) m_{0}>0
$$

and then

$$
(m-1) \pi^{*}\left(K_{X}\right)-\frac{2}{p} E_{m_{0}}^{\prime}-S^{\prime}-S^{\prime \prime} \equiv\left(m-1-\frac{2 m_{0}}{p}\right) \pi^{*}\left(K_{X}\right)
$$

is nef and big, the Kawamata-Viehweg vanishing theorem [17,32] gives a surjective map:

$$
\begin{aligned}
H^{0}\left(X^{\prime}, K_{X^{\prime}}+\left\ulcorner(m-1) \pi^{*}\left(K_{X}\right)-\frac{2}{p} E_{m_{0}}^{\prime}\right\urcorner\right) \\
\longrightarrow H^{0}\left(S^{\prime}, K_{S^{\prime}}+\left.\left\ulcorner(m-1) \pi^{*}\left(K_{X}\right)-\frac{2}{p} E_{m_{0}}^{\prime}\right\urcorner\right|_{S^{\prime}}\right) \\
\oplus H^{0}\left(S^{\prime \prime}, K_{S^{\prime \prime}}+\left.\left\ulcorner(m-1) \pi^{*}\left(K_{X}\right)-\frac{2}{p} E_{m_{0}}^{\prime}\right\urcorner\right|_{S^{\prime \prime}}\right) .
\end{aligned}
$$


The last two groups are non-zero because $p_{g}(X)>0$ (so $p_{g}\left(S^{\prime}\right), p_{g}\left(S^{\prime \prime}\right)>$ $0)$ and $m-1-m_{0}>0$. Therefore $\left|K_{X^{\prime}}+\left\ulcorner(m-1) \pi^{*}\left(K_{X}\right)-\frac{2}{p} E_{m_{0}}^{\prime}\right\urcorner\right|$ can separate $S^{\prime}$ and $S^{\prime \prime}$ and so can $\left|m K_{X^{\prime}}\right|$.

By the birationality principle (P1) and (P2) of [9], it suffices to prove that $\mid m K_{X^{\prime}} \|_{S}$ on $S$ gives a birational map onto its image. Practically we may study a smaller linear system than $\mid m K_{X^{\prime}} \|_{S}$ on $S$. Noting that

$$
(m-1) \pi^{*}\left(K_{X}\right)-\frac{1}{p} E_{m_{0}}^{\prime}-S \equiv\left(m-1-\frac{m_{0}}{p}\right) \pi^{*}\left(K_{X}\right)
$$

is nef and big under the assumptions (iv) or (v), the vanishing theorem gives a surjective map

$$
\begin{aligned}
& H^{0}\left(X^{\prime}, K_{X^{\prime}}+\left\ulcorner(m-1) \pi^{*}\left(K_{X}\right)-\frac{1}{p} E_{m_{0}}^{\prime}\right\urcorner\right) \\
& \quad \longrightarrow H^{0}\left(S, K_{S}+\left.\left\ulcorner(m-1) \pi^{*}\left(K_{X}\right)-S-\frac{1}{p} E_{m_{0}}^{\prime}\right\urcorner\right|_{S}\right) .
\end{aligned}
$$

Note that $\left|K_{X^{\prime}}+\left\ulcorner(m-1) \pi^{*}\left(K_{X}\right)-\frac{1}{p} E_{m_{0}}^{\prime}\right\urcorner\right| \subset\left|m K_{X^{\prime}}\right|$. It suffices to prove that

$$
\left|K_{S}+\left\ulcorner(m-1) \pi^{*}\left(K_{X}\right)-S-\frac{1}{p} E_{m_{0}}^{\prime}\right\urcorner\right|_{S} \mid
$$

gives a birational map.

The birationality principle again allows us to study the restriction to curves by assumption (ii). Now consider a generic irreducible element $C \in$ $|G|$. By assumption (iii), there is an effective $\mathbb{Q}$-divisor $H$ on $S$ such that

$$
\left.\frac{1}{\beta} \pi^{*}\left(K_{X}\right)\right|_{S} \equiv C+H
$$

By the vanishing theorem, whenever $m-1-\frac{m_{0}}{p}-\frac{1}{\beta}>0$, we have the surjective map

$H^{0}\left(S, K_{S}+\left\ulcorner\left.\left((m-1) \pi^{*}\left(K_{X}\right)-S-\frac{1}{p} E_{m_{0}}^{\prime}\right)\right|_{S}-H\right\urcorner\right) \longrightarrow H^{0}\left(C, K_{C}+D\right)$,

where

$$
D:=\left.\left\ulcorner\left.\left((m-1) \pi^{*}\left(K_{X}\right)-S-\frac{1}{p} E_{m_{0}}^{\prime}\right)\right|_{S}-C-H\right\urcorner\right|_{C}
$$

is a divisor on $C$. Noting that

$$
\left.\left((m-1) \pi^{*}\left(K_{X}\right)-S-\frac{1}{p} E_{m_{0}}^{\prime}\right)\right|_{S}-C-\left.H \equiv\left(m-1-\frac{m_{0}}{p}-\frac{1}{\beta}\right) \pi^{*}\left(K_{X}\right)\right|_{S}
$$


and that $C$ is nef on $S$, we have $\operatorname{deg}(D) \geq \alpha$ and thus $\operatorname{deg}(D) \geq \alpha_{0}$. Whenever $C$ is non-hyperelliptic, $m-1-\frac{m_{0}}{p}-\frac{1}{\beta}>0$ and $C$ is an even divisor on $S, \operatorname{deg}(D) \geq 2$ automatically follows and thus $\left|K_{C}+D\right|$ gives a birational map. Whenever $\operatorname{deg}(D) \geq 3$, then

$$
\mid K_{S}+\left\ulcorner\left.\left((m-1) \pi^{*}\left(K_{X}\right)-S-\frac{1}{p} E_{m_{0}}^{\prime}\right)\right|_{S}-H\right\urcorner \|_{C}
$$

gives a birational map. Since

$$
\begin{gathered}
\left|K_{S}+\left\ulcorner\left((m-1) \pi^{*}\left(K_{X}\right)-S-\frac{1}{p} E_{m_{0}}^{\prime}\right) \mid S-H\right\urcorner\right| \\
\subset\left|K_{S}+\left\ulcorner(m-1) \pi^{*}\left(K_{X}\right)-S-\frac{1}{p} E_{m_{0}}^{\prime}\right\urcorner\right|_{S} \mid,
\end{gathered}
$$

the latter linear system gives a birational map. So $\varphi_{m}$ of $X$ is birational.

Finally we show the inequality for $\xi$. Whenever we have $\operatorname{deg}(D) \geq 2$, $\left|K_{C}+D\right|$ is base point free by the curve theory. Denote by $\left|M_{m}\right|$ the movable part of $\left|m K_{X^{\prime}}\right|$ and by $\left|N_{m}\right|$ the movable part of $\mid K_{S}+\left\ulcorner\left((m-1) \pi^{*}\left(K_{X}\right)-\right.\right.$ $\left.\left.S-\frac{1}{p} E_{m_{0}}^{\prime}\right)\left.\right|_{S}-H\right\urcorner$. Applying [9, Lemma 2.7] to surjective maps (3.1) and $(3.2)$, we have

$$
\left.m \pi^{*}\left(K_{X}\right)\right|_{S} \geq N_{m} \quad \text { and } \quad\left(N_{m} \cdot C\right)_{S} \geq 2 g(C)-2+\operatorname{deg}(D) .
$$

Note that the above inequality holds without conditions (i) and (ii). We are done.

Remark 3.2. If we replace $M_{m_{0}}$ in Theorem 3.1 by any divisor $N_{m_{0}} \leq M_{m_{0}}$ with $h^{0}\left(X^{\prime}, N_{m_{0}}\right) \geq 2$, Theorem 3.1 is still true accordingly. This is clear by the proof. The main point is that it suffices to prove that a sub-linear system of $\left|m K_{X^{\prime}}\right|$ gives a birational map. To avoid frustrating setting up and more complicated notations, we omit the proof in details. The idea is, however, trivially similar.

While applying Theorem 3.1, one has to choose a suitable movable system $|G|$ on $S$. Then quite a technical problem is to find a suitable $\beta$ as in Theorem 3.1(iii). The following lemma presents the way for the most difficult case - the rational pencil case.

Lemma 3.3. Keep the same notation as in Section 2.1 and Theorem 3.1. Assume $B=\mathbb{P}^{1}$. Let $f: X^{\prime} \longrightarrow \mathbb{P}^{1}$ be an induced fibration of $\varphi_{m_{0}}$. Denote by 
$F:=S$ a general fiber of $f$. Then one can find a sequence of rational numbers $\left\{\beta_{n}\right\}$ with $\lim _{n \mapsto+\infty} \beta_{n}=p /\left(m_{0}+p\right)$ such that $\left.\pi^{*}\left(K_{X}\right)\right|_{F}-\beta_{n} \sigma^{*}\left(K_{F_{0}}\right)$ is $\mathbb{Q}$-linearly equivalent to an effective $\mathbb{Q}$-divisor $N_{n}$, where $\sigma: F \longrightarrow F_{0}$ is the blow down onto the smooth minimal model.

Proof. One has $\mathcal{O}_{B}(p) \hookrightarrow f_{*} \omega_{X^{\prime}}^{m_{0}}$ and therefore $f_{*} \omega_{X^{\prime} / B}^{t_{0} p} \hookrightarrow f_{*} \omega_{X^{\prime}}^{t_{0} p+2 t_{0} m_{0}}$ for any big integer $t_{0}$.

For any positive integer $k$, denote by $M_{k}$ the movable part of $\left|k K_{X^{\prime}}\right|$. Note that $f_{*} \omega_{X^{\prime} / B}^{t_{0} p}$ is generated by global sections since it is semi-positive according to Viehweg [33]. So any local section can be extended to a global one. On the other hand, $\left|t_{0} p \sigma^{*}\left(K_{F_{0}}\right)\right|$ is base point free and is exactly the movable part of $\left|t_{0} p K_{F}\right|$ by Bombieri [2] or Reider [25]. Clearly one has the following relation:

$$
\left.a_{0} \pi^{*}\left(K_{X}\right)\right|_{F} \geq\left. M_{t_{0} p+2 t_{0} m_{0}}\right|_{F} \geq b_{0} \sigma^{*}\left(K_{F_{0}}\right)
$$

where $a_{0}:=t_{0} p+2 t_{0} m_{0}$ and $b_{0}:=t_{0} p$. This means that there is an effective $\mathbb{Q}$-divisor $E_{0}^{\prime}$ on $F$ such that

$$
\left.a_{0} \pi^{*}\left(K_{X}\right)\right|_{F}={ }_{\mathbb{Q}} b_{0} \sigma^{*}\left(K_{F_{0}}\right)+E_{0}^{\prime} .
$$

Thus $\left.\pi^{*}\left(K_{X}\right)\right|_{F}=\mathbb{Q}\left(p /\left(p+2 m_{0}\right)\right) \sigma^{*}\left(K_{F_{0}}\right)+E_{0}$ with $E_{0}=\frac{1}{a_{0}} E_{0}^{\prime}$.

We consider the case $p \geq 2$.

Assume that we have defined $a_{n}$ and $b_{n}$ such that the following is satisfied with $l=n$ :

$$
\left.a_{l} \pi^{*}\left(K_{X}\right)\right|_{F} \geq b_{l} \sigma^{*}\left(K_{F_{0}}\right) .
$$

We will define $a_{n+1}$ and $b_{n+1}$ inductively such that the above inequality is satisfied with $l=n+1$. One may assume from the beginning that $a_{n} \pi^{*}\left(K_{X}\right)$ supports on a divisor with normal crossings. Then the Kawamata-Viehweg vanishing theorem implies the surjective map

$$
H^{0}\left(K_{X^{\prime}}+\left\ulcorner a_{n} \pi^{*}\left(K_{X}\right)\right\urcorner+F\right) \longrightarrow H^{0}\left(F, K_{F}+\left.\left\ulcorner a_{n} \pi^{*}\left(K_{X}\right)\right\urcorner\right|_{F}\right) .
$$

One has the relation

$$
\begin{aligned}
\mid K_{X^{\prime}}+\left\ulcorner a_{n} \pi^{*}\left(K_{X}\right)\right\urcorner+F \|_{F} & =\left|K_{F}+\left\ulcorner a_{n} \pi^{*}\left(K_{X}\right)\right\urcorner\right|_{F} \mid \\
& \supset\left|K_{F}+b_{n} \sigma^{*}\left(K_{F_{0}}\right)\right| \\
& \supset\left|\left(b_{n}+1\right) \sigma^{*}\left(K_{F_{0}}\right)\right| .
\end{aligned}
$$

Denote by $M_{a_{n}+1}^{\prime}$ the movable part of $\left|\left(a_{n}+1\right) K_{X^{\prime}}+F\right|$. Applying [9, Lemma 2.7] again, one has $\left.M_{a_{n}+1}^{\prime}\right|_{F} \geq\left(b_{n}+1\right) \sigma^{*}\left(K_{F_{0}}\right)$. Re-modifying 
our original $\pi$ such that $\left|M_{a_{n}+1}^{\prime}\right|$ is base point free. In particular, $M_{a_{n}+1}^{\prime}$ is nef. Since $X$ is of general type $\left|m K_{X}\right|$ gives a birational map whenever $m$ is big enough. Thus we see that $M_{a_{n}+1}^{\prime}$ is big if we fix a very big $t_{0}$ in advance.

Now the Kawamata-Viehweg vanishing theorem again gives

$$
\begin{aligned}
\mid K_{X^{\prime}}+M_{a_{n}+1}^{\prime}+F \|_{F} & =\left|K_{F}+M_{a_{n}+1}^{\prime}\right| F \mid \\
& \supset\left|K_{F}+\left(b_{n}+1\right) \sigma^{*}\left(K_{F_{0}}\right)\right| \\
& \supset\left|\left(b_{n}+2\right) \sigma^{*}\left(K_{F_{0}}\right)\right| .
\end{aligned}
$$

We may repeat the above procedure inductively. Denote by $M_{a_{n}+t}^{\prime}$ the movable part of $\left|K_{X^{\prime}}+M_{a_{n}+t-1}^{\prime}+F\right|$ for $t \geq 2$. For the same reason, we may assume $\left|M_{a_{n}+t}^{\prime}\right|$ to be base point free. Inductively one has:

$$
\left.M_{a_{n}+t}^{\prime}\right|_{F} \geq\left(b_{n}+t\right) \sigma^{*}\left(K_{F_{0}}\right) \text {. }
$$

Applying the vanishing theorem once more, we have

$$
\begin{aligned}
\mid K_{X^{\prime}}+M_{a_{n}+t}^{\prime}+F \|_{F} & =\left|K_{F}+M_{a_{n}+t}^{\prime}\right| F \mid \\
& \supset\left|K_{F}+\left(b_{n}+t\right) \sigma^{*}\left(K_{F_{0}}\right)\right| \\
& \supset\left|\left(b_{n}+t+1\right) \sigma^{*}\left(K_{F_{0}}\right)\right| .
\end{aligned}
$$

Take $t=p-1$. Noting that

$$
\left|K_{X^{\prime}}+M_{a_{n}+p-1}^{\prime}+F\right| \subset\left|\left(a_{n}+p+m_{0}\right) K_{X^{\prime}}\right|
$$

and applying [9, Lemma 2.7] again, one has

$$
\left.a_{n+1} \pi^{*}\left(K_{X}\right)\right|_{F} \geq\left. M_{a_{n}+p+m_{0}}\right|_{F} \geq\left. M_{a_{n}+p}^{\prime}\right|_{F} \geq b_{n+1} \sigma^{*}\left(K_{F_{0}}\right) .
$$

Here we set $a_{n+1}:=a_{n}+p+m_{0}$ and $b_{n+1}=b_{n}+p$. Set $\beta_{n}=\frac{b_{n}}{a_{n}}$. Clearly $\lim _{n \mapsto+\infty} \beta_{n}=p /\left(m_{0}+p\right)$.

The case $p=1$ can be proved similarly, but with a simpler induction. We omit the details.

The following Lemma is needed in our proof. Though similar one has already been established in several papers of the first author, we include it here for the convenience to readers.

Lemma 3.4. Keep the same notation as in Section 2.1. Let $f: X^{\prime} \longrightarrow B$ be the induced fibration of $\varphi_{m_{0}}$. Denote by $F:=S$ a general fiber of $f$. If $\operatorname{dim}(B)=1$ and $g(B)>0$, then $\left.\pi^{*}\left(K_{X}\right)\right|_{F} \sim \sigma^{*}\left(K_{F_{0}}\right)$ where $\sigma: F \longrightarrow F_{0}$ is the blow down onto the smooth minimal model. 
Proof. We shall use the idea of Lemma 14 in Kawamata's paper [18]. By Shokurov's theorem in [26](see also [14]), each fiber of $\pi: X^{\prime} \longrightarrow X$ is rationally chain connected. Therefore, $f\left(\pi^{-1}(x)\right)$ is a point for all $x \in X$. Considering the image $G \subset(X \times B)$ of $X^{\prime}$ via the morphism $(\pi \times f) \circ \triangle_{X^{\prime}}$, where $\triangle_{X^{\prime}}$ is the diagonal map $X^{\prime} \longrightarrow X^{\prime} \times X^{\prime}$, one knows that $G$ is a projective variety. Let $g_{1}: G \longrightarrow X$ and $g_{2}: G \longrightarrow B$ be two projections. Since $g_{1}$ is a projective morphism and even a bijective map, $g_{1}$ must be both a finite morphism of degree 1 and a birational morphism. Since $X$ is normal, $g_{1}$ must be an isomorphism. So $f$ factors as $f_{1} \circ \pi$, where $f_{1}:=g_{2} \circ g_{1}^{-1}: X \rightarrow B$ is a well-defined morphisms. In particular, a general fiber $F_{0}$ of $f_{1}$ must be smooth minimal. So it is clear that $\left.\pi^{*}\left(K_{X}\right)\right|_{F} \sim \sigma^{*}\left(K_{F_{0}}\right)$, where $\sigma$ is nothing but $\left.\pi\right|_{F}$.

\section{Proof of the main theorem}

We begin to prove Theorem 1.1. Let $X$ be a complex minimal projective 3 -fold of general type with $\mathbb{Q}$-factorial terminal singularities and $\chi\left(\omega_{X}\right) \geq 0$.

\subsection{Reduction to the case $p_{g}=1$}

If $p_{g}(X)>1$, then $\varphi_{8}$ is birational by [8]. If $q(X)>0$, then $\varphi_{m}$ is birational for all $m \geq 7$ by [6]. Thus we assume, from now on, that $p_{g}(X) \leq 1$ and $q(X)=0$. The assumption

$$
0 \leq \chi\left(\omega_{X}\right)=-\chi\left(\mathcal{O}_{X}\right)=-1+q(X)-h^{2}\left(\mathcal{O}_{X}\right)+p_{g}(X)
$$

implies $p_{g}(X) \geq h^{2}\left(\mathcal{O}_{X}\right)+1 \geq 1$. Then we clearly have $p_{g}(X)=1$, $h^{2}\left(\mathcal{O}_{X}\right)=0$ and $\chi\left(\omega_{X}\right)=0$.

According to [4], we only have to study non-Gorenstein minimal 3-folds $X$. In practice, we may assume the Cartier index $r(X)>1$.

\subsection{Plurigenus}

Let $X$ be a minimal 3-fold of general type with terminal singularities. Recall Reid's plurigenus formula ([23, p. 413]):

$$
P_{m}(X)=\frac{1}{12} m(m-1)(2 m-1) K_{X}^{3}-(2 m-1) \chi\left(\mathcal{O}_{X}\right)+\sum_{Q} R_{m}(Q)
$$


where $m>1$ is an integer, the correction term

$$
R_{m}(Q):=\frac{r^{2}-1}{12 r}(m-\bar{m})+\sum_{j=0}^{\bar{m}-1} \frac{\overline{b j}(r-\overline{b j})}{2 r}
$$

and the sum $\sum_{Q}$ runs through all baskets of singularities $Q$ of type $\frac{1}{r}(a,-a, 1)$ with the positive integer $a$ coprime to $r, 0<a<r, 0<b<r, a b \equiv 1(\bmod r)$ and $\bar{m}$ the smallest residue of $\mathrm{m} \bmod r$. Reid's result ([23, Theorem 10.2]) says that the above baskets $\{Q\}$ of singularities are in fact virtual (!) though $X$ may have non-quotient terminal singularities. Iano-Fletcher ([15]) actually shows that the set of baskets $\{Q\}$ in Reid's formula is uniquely determined by $X$.

Lemma 4.1. For all basket $Q, R_{5}(Q) \geq R_{4}(Q) \geq R_{3}(Q) \geq R_{2}(Q)$. In particular,

$$
P_{5}(X)>P_{4}(X)>P_{3}(X)>P_{2}(X) \geq 1
$$

for all 3-fold $X$ with $\chi\left(\mathcal{O}_{X}\right)=0$.

Proof. Suppose that $Q$ is of type $\frac{1}{r}(a,-a, 1)=\frac{1}{r}(1,-1, b)$ with $r>1, a$ coprime to $r, a b \equiv 1(\bmod \mathrm{r})$ and $0<b<r$.

When $r=2$, one has

$$
R_{3}(Q)=\frac{r^{2}-1}{6 r}=\frac{1}{4}=R_{2}(Q) .
$$

When $r=3$, one has

$$
R_{3}(Q)=\frac{r^{2}-1}{4 r}=\frac{2}{3}>\frac{1}{3}=\frac{b(r-b)}{2 r}=R_{2}(Q) .
$$

When $r>3$, one has $m=\bar{m}$ for $m=2,3$ and

$$
R_{3}(Q)=\sum_{j=0}^{2} \frac{\overline{b j}(r-\overline{b j})}{2 r} \geq \sum_{j=0}^{1} \frac{\overline{b j}(r-\overline{b j})}{2 r}=R_{2}(Q) .
$$

If $\chi\left(\mathcal{O}_{X}\right)=0$, then Reid's formula (4.1) gives

$$
P_{3}(X)=\frac{5}{2} K_{X}^{3}+\sum_{Q} R_{3}(Q)>\frac{1}{2} K_{X}^{3}+\sum_{Q} R_{2}(Q)=P_{2}(X)>0 .
$$

In particular, $P_{3}(X) \geq 2$. 
Similarly one can verify the inequality $P_{5}(X)>P_{4}(X)>P_{3}(X)$. So $P_{5}(X) \geq 4$.

Now Theorem 1.1 essentially follows from the following theorem by letting $m_{0}=2,3$.

Theorem 4.2. Let $X$ be a minimal projective 3-fold of general type with $\mathbb{Q}$-factorial terminal singularities, $p_{g}(X)=1$ and $P_{m_{0}}(X) \geq 2$. Then $\varphi_{m}$ is birational onto its image under one of the following conditions:

(1) $m \geq 4 m_{0}+3$ and $2 \leq m_{0} \leq 3$;

(2) $m \geq 4 m_{0}+2$ and $m_{0} \geq 4$;

(3) $m \geq 14, \chi\left(\mathcal{O}_{X}\right)=0$ and $m_{0}=3$.

Proof. Set $d_{m_{0}}:=\operatorname{dim} \varphi_{m_{0}}(X)$. We discuss according to the value of $d_{m_{0}}$. We shall mainly apply Theorem 3.1. Because $p_{g}(X)>0,3.1(\mathrm{i})$ is always satisfied whenever $m \geq m_{0}+2$.

Case 1. $d_{m_{0}}=3$. Recall that $S$ is a generic irreducible element of $\left|M_{m_{0}}\right|$. We have $p=1$. On $S$ we take $G:=\left.S\right|_{S}$. Clearly $|G|$ is base point free and is not composed of a pencil. So a generic irreducible element $C$ of $|G|$ is a smooth curve. Also under the assumption $m \geq m_{0}+2$ one has

$$
\begin{aligned}
& K_{S}+\left.\left\ulcorner(m-1) \pi^{*}\left(K_{X}\right)-S-E_{m_{0}}^{\prime}\right\urcorner\right|_{S} \\
& \quad \geq\left(\left.K_{X^{\prime}}\right|_{S}+\left.\left\ulcorner(m-1) \pi^{*}\left(K_{X}\right)-S-E_{m_{0}}^{\prime}\right\urcorner\right|_{S}\right)+G \geq G .
\end{aligned}
$$

Thus 3.1(ii) is also satisfied. Because $\left.m_{0} \pi^{*}\left(K_{X}\right)\right|_{S} \geq C$, we may take $\beta=\frac{1}{m_{0}}$ and so (iii) is satisfied.

Note that $C^{2} \geq 2$ because $|G|$ is not composed of a pencil. So $m_{0} \pi^{*}$ $\left.\left(K_{X}\right)\right|_{S} \cdot C \geq C^{2} \geq 2$, which implies $\xi \geq \frac{2}{m_{0}}$. Now we take $m \geq 3 m_{0}+2$ and run Theorem 3.1. One has $\alpha=\left(m-1-m_{0} / p-1 / \beta\right) \xi \geq 2+2 / m_{0}>2$. This means that $\varphi_{m}$ is birational for all $m \geq 3 m_{0}+2$. This is not the best. In fact, Theorem 3.1 already gives $\xi \geq(2 g(C)+1) /\left(3 m_{0}+2\right)$. Note that $2 g(C)-2=\left(K_{S}+C\right) \cdot C=\left(\left.K_{X^{\prime}}\right|_{S}+2 C\right) \cdot C>4$. One has $\xi \geq 9 /\left(3 m_{0}+2\right)$. Now take $m>\frac{8}{3} m_{0}+\frac{13}{9}$. One has $\alpha=\left(m-1-2 m_{0}\right) \xi>2$. Theorem 3.1 says that $\varphi_{m}$ is birational whenever $m>\frac{8}{3} m_{0}+\frac{13}{9}$. (One may go on optimizing the estimate. We stop here since we have already proved the theorem.)

Case 2. $\quad d_{m_{0}}=2$. We have $p=1$. On $S$ we take $G:=\left.S\right|_{S}$. Clearly $|G|$ is base point free and is composed of a pencil. So a generic irreducible element $C$ of $|G|$ is a smooth curve. One has $G \equiv t C$ for $t \geq P_{m_{0}}-2 \geq 1$. 
Also under the assumption $m \geq m_{0}+2$ one has

$$
\begin{aligned}
& K_{S}+\left.\left\ulcorner(m-1) \pi^{*}\left(K_{X}\right)-S-E_{m_{0}}^{\prime}\right\urcorner\right|_{S} \\
& \quad \geq\left(\left.K_{X^{\prime}}\right|_{S}+\left.\left\ulcorner(m-1) \pi^{*}\left(K_{X}\right)-S-E_{m_{0}}^{\prime}\right\urcorner\right|_{S}\right)+G \geq C .
\end{aligned}
$$

So $\left|K_{S}+\left\ulcorner(m-1) \pi^{*}\left(K_{X}\right)-S-E_{m_{0}}^{\prime}\right\urcorner\right|_{S} \mid$ can separate different generic irreducible elements of $|G|$ provided that $|G|$ is composed of a rational pencil. When $|G|$ is composed of an irrational pencil, we need the assumption $m \geq 2 m_{0}+2$. In fact, we have $\left.S\right|_{S} \equiv t C$ with $t \geq 2$ and $\left.m_{0} \pi^{*}\left(K_{X}\right)\right|_{S}$ $\equiv t C+\left.E_{m_{0}}^{\prime}\right|_{S}$. Take two generic irreducible elements $C^{\prime}, C^{\prime \prime}$ of $|G|$. Because

$$
\left.\left(m-m_{0}-1\right) \pi^{*}\left(K_{X}\right)\right|_{S}-C^{\prime}-C^{\prime \prime}-\left.\frac{2}{t} E_{m_{0}}^{\prime} \equiv\left(m-m_{0}-\frac{2}{t} m_{0}-1\right) \pi^{*}\left(K_{X}\right)\right|_{S}
$$

is nef and big, the Kawamata-Viehweg vanishing theorem gives a surjective map

$$
\begin{gathered}
H^{0}\left(S, K_{S}+\left\ulcorner\left.\left(m-m_{0}-1\right) \pi^{*}\left(K_{X}\right)\right|_{S}-\left.\frac{2}{t} E_{m_{0}}^{\prime}\right|_{S}\right\urcorner\right) \\
\longrightarrow H^{0}\left(C^{\prime}, K_{C^{\prime}}+D^{\prime}\right) \oplus H^{0}\left(C^{\prime \prime}, K_{C^{\prime \prime}}+D^{\prime \prime}\right),
\end{gathered}
$$

where $D^{\prime}, D^{\prime \prime}$ are divisors of positive degree. Besides, the last groups are non-zero. Noting that

$$
\begin{aligned}
& K_{S}+\left.\left\ulcorner(m-1) \pi^{*}\left(K_{X}\right)-S-E_{m_{0}}^{\prime}\right\urcorner\right|_{S} \geq K_{S}+\left\ulcorner\left.\left(m-m_{0}-1\right) \pi^{*}\left(K_{X}\right)\right|_{S}\right\urcorner \\
& \quad \geq K_{S}+\left\ulcorner\left.\left(m-m_{0}-1\right) \pi^{*}\left(K_{X}\right)\right|_{S}-\frac{2}{t} E_{m_{0}}^{\prime} \mid S\right\urcorner,
\end{aligned}
$$

$\left|K_{S}+\left\ulcorner(m-1) \pi^{*}\left(K_{X}\right)-S-E_{m_{0}}^{\prime}\right\urcorner\right|_{S} \mid$ separates $C^{\prime}$ and $C^{\prime \prime}$. So we see that 3.1(ii) is satisfied whenever $m \geq 2 m_{0}+2$.

Because $\left.m_{0} \pi^{*}\left(K_{X}\right)\right|_{S} \geq C$, we may take $\beta=\frac{1}{m_{0}}$ and so (iii) is satisfied. If we take a big $m$ such that $\alpha$ is big enough, then Theorem 3.1 gives

$$
m \xi \geq 2 g(C)-2+\left(m-1-\frac{m_{0}}{p}-\frac{1}{\beta}\right)
$$

which says $\xi \geq 2 /\left(2 m_{0}+1\right)$. This is only an initial estimate. Take $m>$ $4 m_{0}+2$. Then $\alpha=\left(m-2 m_{0}-1\right) \xi>2$. Theorem 3.1 says that $\varphi_{m}$ is birational and that $\xi \geq 5 /\left(4 m_{0}+3\right)$. Take $m=3 m_{0}+2$. Then $\alpha=(m-$ $\left.2 m_{0}-1\right) \xi>1$. Theorem 3.1 gives $\xi \geq 4 /\left(3 m_{0}+2\right)$. Finally take $m>$ 
$\frac{7}{2} m_{0}+2$. Then $\alpha=\left(m-2 m_{0}-1\right) \xi>2$. Theorem 3.1 says that $\varphi_{m}$ is birational whenever $m>\frac{7}{2} m_{0}+2$.

Case 3. $\quad d_{m_{0}}=1$. We have an induced fibration $f: X^{\prime} \longrightarrow B$. Denote by $F:=S$ a general fiber of $f$. Note that $F$ is a surface of general type. Denote by $\sigma: F \longrightarrow F_{0}$ the blowing down onto the minimal model.

Subcase 3.1. $g(B)>0$. By Lemma 3.4, we have $\left.\pi^{*}\left(K_{X}\right)\right|_{F} \sim \sigma^{*}\left(K_{F_{0}}\right)$. We need to study the condition (ii) in Theorem 3.1. For all $m \geq m_{0}+5$ one has

$$
\begin{aligned}
K_{F}+\left.\left\ulcorner(m-1) \pi^{*}\left(K_{X}\right)-F-\frac{1}{p} E_{m_{0}}^{\prime}\right\urcorner\right|_{F} & \geq K_{F}+\left\ulcorner\left.\left(m-m_{0}-1\right) \pi^{*}\left(K_{X}\right)\right|_{F}\right\urcorner \\
& \geq K_{F}+\left.\left(m-m_{0}-1\right) \pi^{*}\left(K_{X}\right)\right|_{F} \\
& \geq\left.\left(m-m_{0}\right) \pi^{*}\left(K_{X}\right)\right|_{F} \geq 5 \sigma^{*}\left(K_{F_{0}}\right) .
\end{aligned}
$$

So $\Phi_{\left|K_{F}+\left\ulcorner(m-1) \pi^{*}\left(K_{X}\right)-F-\frac{1}{p} E_{m_{0}}^{\prime}\right\urcorner\right|_{F} \mid}$ already gives a birational map. The proof of Theorem 3.1 tells that we may omit the conditions (iii) $\sim(\mathrm{v})$ in Theorem 3.1. Thus $\varphi_{m}$ is birational for all $m \geq m_{0}+5$.

Subcase 3.2. $g(B)=0$. For a general $F$ the natural map

$$
H^{0}\left(X^{\prime}, K_{X^{\prime}}-F\right) \longrightarrow H^{0}\left(X^{\prime}, K_{X^{\prime}}\right)
$$

is a strict inclusion simply because $F$ is movable. This means that $p_{g}(F)>0$ since $p_{g}\left(X^{\prime}\right)=p_{g}(X)>0$. For our purpose, we classify $F$ into the following three types:

(I) $\left(K_{F_{0}}^{2}, p_{g}(F)=(1,2)\right.$;

(II) $\left(K_{F_{0}}^{2}, p_{g}(F)=(2,3)\right.$;

(III) $F$ does not belong to types (I) and (II).

We first study the type (III) case. By the results of Bombieri [2], Reider [25], Catanese-Ciliberto [3] and Francia [11], $\left|2 \sigma^{*}\left(K_{F_{0}}\right)\right|$ is always base point free whenever $p_{g}(F)>0$. We set $G:=2 \sigma^{*}\left(K_{F_{0}}\right)$ to run Theorem 3.1. The inclusion $\mathcal{O}(1) \hookrightarrow f_{*} \omega_{X^{\prime}}^{m_{0}}$ implies the inclusion

$$
f_{*} \omega_{X^{\prime} / \mathbb{P}^{1}}^{2} \hookrightarrow f_{*} \omega_{X^{\prime}}^{4 m_{0}+2} .
$$

Viehweg [33] first showed that $f_{*} \omega_{X^{\prime} / \mathbb{P}^{1}}^{2}$ is semi-positive. So it is also generated by global sections. Thus it is clear that

$$
\left|M_{4 m_{0}+2}\right|_{F} \supset\left|2 \sigma^{*}\left(K_{F_{0}}\right)\right|
$$


where $M_{4 m_{0}+2}$ is the movable part of $\left|\left(4 m_{0}+2\right) K_{X^{\prime}}\right|$.

(\#) So 3.1(ii) is satisfied for all $m \geq 4 m_{0}+2$ and for all $F$ with $p_{g}(F)>0, G \leq 2 \sigma^{*}\left(K_{F_{0}}\right)$ and $|G|$ not an irrational pencil.

In type (III) case, $G$ is an even divisor under our setting and is not composed of a pencil of curves. On the other hand, the birationality of $\Phi_{\left|3 K_{F}\right|}$ implies that a general $C \in|G|$ is non-hyperelliptic. By Lemma 3.3, we can take a $\beta \mapsto 1 /\left(2 m_{0}+2\right)$ such that $\left.\pi^{*}\left(K_{X}\right)\right|_{F}-\beta C$ is numerically equivalent to an effective $\mathbb{Q}$-divisor. By our definition, one has $p \geq P_{m_{0}}-1 \geq 1$. For all $m \geq 3 m_{0}+4, \alpha=\left(m-1-m_{0} / p-1 / \beta\right) \xi>0$. In general, whenever $m \geq$ $4 m_{0}+2$, Theorem 3.1 asserts that $\varphi_{m}$ is birational.

Next we study the type (II) case. We have $\left(K_{F_{0}}^{2}, p_{g}(F)\right)=(2,3)$. By [1, p. 227], we know that $\left|\sigma^{*}\left(K_{F_{0}}\right)\right|$ is base point free and $\Phi_{\left|K_{F}\right|}$ is finite of degree 2. We set $G:=\sigma^{*}\left(K_{F_{0}}\right)$. Then $C$, as a generic irreducible element of $G$, is smooth and of genus 3 . We have already showed (statement (\#) in type (III) case) that 3.1(ii) is satisfied for all $m \geq 4 m_{0}+2$ because $G \leq 2 \sigma^{*}\left(K_{F_{0}}\right)$. By Lemma 3.3 we can take a $\beta \mapsto 1 /\left(m_{0}+1\right)$ such that $\left.\pi^{*}\left(K_{X}\right)\right|_{F}-\beta C$ is numerically equivalent to an effective $\mathbb{Q}$-divisor. So $\xi=\left.\pi^{*}\left(K_{X}\right)\right|_{F} \cdot C \geq$ $\beta C^{2} \mapsto 2 /\left(m_{0}+1\right)$. Taking the limit one has $\xi \geq 2 /\left(m_{0}+1\right)$. Similarly we have $p \geq 1$. Take $m=3 m_{0}+2$. Then $\alpha=\left(m-1-m_{0} / p-1 / \beta\right) \xi \geq$ $2 m_{0} /\left(m_{0}+1\right)>1$. Theorem 3.1 gives $\xi \geq 6 /\left(3 m_{0}+2\right)$. In order to get the birationality, we need the assumption $m \geq 4 m_{0}+2$, under which one has

$$
\alpha=\left(m-1-\frac{m_{0}}{p}-\frac{1}{\beta}\right) \xi \geq \frac{12 m_{0}}{3 m_{0}+2}>2 .
$$

Thus $\varphi_{m}$ is birational whenever $m \geq 4 m_{0}+2$.

Finally we study the type (I) case. We have $\left(K_{F_{0}}^{2}, p_{g}(F)\right)=(1,2)$. By [1] we know that the movable part of $\left|K_{F}\right|$ has one simple base point. Take $|G|$ to be the movable part of $\left|K_{F}\right|$. Then a generic irreducible element $C$ of $|G|$ is a smooth curve of genus 2. Similarly we need the assumption $m \geq 4 m_{0}+2$ to secure the condition 3.1(ii) (by virtue of the statement (\#) in the type (III) case because $|G|$ is a rational pencil). By Lemma 3.3 we can take a $\beta \mapsto 1 /\left(m_{0}+1\right)$ such that $\left.\pi^{*}\left(K_{X}\right)\right|_{F}-\beta C$ is numerically equivalent to an effective $\mathbb{Q}$-divisor. Clearly we have $p \geq 1$. If we take a big $m$ such that $\alpha$ is big enough, Theorem 3.1 gives

$$
m \xi \geq 2 g(C)-2+\left(m-2 m_{0}-2\right) \xi
$$


which implies $\xi \geq 1 /\left(m_{0}+1\right)$. Take $m=4 m_{0}+5$. Then $\alpha \geq\left(2 m_{0}+3\right) /$ $\left(m_{0}+1\right)>2$. Theorem 3.1 gives $\xi \geq 5 /\left(4 m_{0}+5\right)$ and so does the birationality of $\varphi_{m}$. Take $m=4 m_{0}+4$. Then $\alpha \geq\left(10 m_{0}+10\right) /\left(4 m_{0}+5\right)>2$. One has the birationality of $\varphi_{m}$ and $\xi \geq 5 /\left(4 m_{0}+4\right)$. Take $m=4 m_{0}+3$. Then $\alpha \geq\left(10 m_{0}+5\right) /\left(4 m_{0}+4\right)>2$ whenever $m_{0} \geq 2$. So $\varphi_{4 m_{0}+3}$ is birational and $\xi \geq 5 /\left(4 m_{0}+3\right)$. We have already proved Theorem $4.2(1)$.

Assume $m_{0} \geq 4$ and take $m=4 m_{0}+2$. Then $\alpha \geq 10 m_{0} /\left(4 m_{0}+3\right)>2$. Theorem 3.1 gives the birationality of $\varphi_{4 m_{0}+2}$. We have proved Theorem $4.2(2)$.

The only left case to verify is $m_{0}=3$ and $\chi\left(\mathcal{O}_{X}\right)=0$ for which we have already the birationality of $\varphi_{4 m_{0}+3}$. We have

$$
\xi \geq \frac{1}{3}
$$

as shown above.

(**) Furthermore the assumption $m_{0}=3, \chi\left(\mathcal{O}_{X}\right)=0$ and $\xi>\frac{1}{3}$ gives $\alpha \geq 2 m_{0} \xi>2$, which means that $\varphi_{4 m_{0}+2}=\varphi_{14}$ is birational by Theorem 3.1.

Claim 4.3. When $m_{0}=3$ and $\chi\left(\mathcal{O}_{X}\right)=0, \varphi_{4 m_{0}+2}=\varphi_{14}$ is birational.

To prove the claim, we need to study the 5 -canonical system $\left|5 K_{X^{\prime}}\right|$. By Lemma 4.1 , one has $P_{5}(X) \geq 4$. Denote $d_{5}:=\operatorname{dim} \varphi_{5}(X)$. Keep the same notation as in Section 2.1 and in Theorem 3.1. Recall that $M_{5}$ is the movable part of $\left|5 K_{X^{\prime}}\right|$. The induced fibration from $\varphi_{5}$ is denoted by $f_{5}: X^{\prime} \longrightarrow W_{5}$. (In fact, we can take further modifications over $X^{\prime}$ (still denote by $X^{\prime}$ the final modification) such that the final $X^{\prime}$ dominates both $\varphi_{3}$ and $\varphi_{5}$.)

If $d_{5}=3$, then $\operatorname{dim} \varphi_{5}(F)=2$ and $\operatorname{dim} \varphi_{5}(C)=1$ for a general curve $C \in|G|$ in a general fiber $F$. This means that the linear system $\mid M_{5} \|_{C}(\subset$ $\left.\left|M_{5}\right| C \mid\right)$ gives a finite map from $C$ onto a curve and so does $\left|M_{5}\right|_{C} \mid$. The Riemann-Roch and Clifford theorem on $C$ says that $5 \pi^{*}\left(K_{X}\right) \cdot C \geq M_{5}$. $C \geq 2$, i.e., $\xi \geq \frac{2}{5}>\frac{1}{3}$. Statement $(* *)$ tells that $\varphi_{14}$ is birational.

If $d_{5}=1$, then $\left|5 K_{X^{\prime}}\right|$ induces the same fibration $f: X^{\prime} \longrightarrow W_{5}=B$ simply because $5 K_{X^{\prime}} \geq 3 K_{X^{\prime}}$. The typical property here is that $5 \pi^{*}\left(K_{X}\right) \geq$ $3 F$ for a general fiber $F$. By Lemma 3.3 (just take $m_{0}^{\prime}=5$ and $p^{\prime}=3$ ) we can find a $\beta \mapsto \frac{3}{8}$ such that $\left.\pi^{*}\left(K_{X}\right)\right|_{F}-\beta C$ is numerically equivalent to an effective $\mathbb{Q}$-divisor. Going on the argument just before the claim, we have $m_{0}=3, p=1, \beta=\frac{3}{8}$ and $\xi \geq \frac{1}{3}$ as in (4.2). Take $m=14$. Then $\alpha=\left(m-1-m_{0}-\frac{1}{\beta}\right) \xi>2$. So Theorem 3.1 gives the birationality of $\varphi_{14}$. 
Finally if $d_{5}=2$, we need to study the image surface $W_{5}^{\prime}$ of $X^{\prime}$ through the morphism $\Phi_{\left|M_{5}\right|}$. In fact, we have the decomposition

$$
\Phi_{\left|M_{5}\right|}: X^{\prime} \stackrel{f_{5}}{\longrightarrow} W_{5} \stackrel{s_{5}}{\longrightarrow} W_{5}^{\prime} \subset \mathbb{P}^{P_{5}(X)-1} .
$$

There is a very ample divisor $H_{5}$ on $W_{5}^{\prime}$ such that $M_{5}=\Phi_{\left|M_{5}\right|}^{*}\left(H_{5}\right)$. Furthermore one has $\left.M_{5}\right|_{S_{5}} \equiv a_{5} \tilde{C}$ for a general member $S_{5} \in\left|M_{5}\right|$ and an integer $a_{5} \geq \operatorname{deg} s_{5} \operatorname{deg}\left(W_{5}^{\prime}\right) \geq \operatorname{deg}\left(W_{5}^{\prime}\right) \geq P_{5}(X)-2, \tilde{C}$ is a general fiber of $f_{5}$. If $a_{5} \geq 3$, we may utilize our argument in Case 2 replacing $m_{0}$ by $m_{0}^{\prime}=5$. Over there we have shown $\xi \geq 4 /\left(3 m_{0}^{\prime}+2\right)=\frac{4}{17}$. But we can take a better $\beta$, namely $\beta=\frac{3}{5}$. We have $p=1$. Take $m=12$. Then $\alpha=$ $(12-1-5-(5 / 3)) \xi>1$. Theorem 3.1 gives $\xi \geq \frac{1}{3}$. Take $m=14$. Then $\alpha=(14-1-5-(5 / 3)) \xi \geq \frac{19}{9}>2$. Theorem 3.1 says that $\varphi_{14}$ is birational.

So we are left to study what happens if $a_{5}=2$. This means that $\operatorname{deg}\left(W_{5}^{\prime}\right)=2, P_{5}(X)=4$ and $\operatorname{deg}\left(s_{5}\right)=1$. Recall that a degree 2 irreducible surface in $\mathbb{P}^{3}$ must be one of the following type of surfaces (see, for instance, Reid's lecture notes [24, Exercise 19, p. 30]):

(a) $W_{5}^{\prime}$ is the cone $\overline{\mathbb{F}}_{2}$ by blowing down the unique section with the selfintersection -2 on the Hirzebruch surface $\mathbb{F}_{2}$ (a ruled surface);

(b) $W_{5}^{\prime}=\mathbb{P}^{1} \times \mathbb{P}^{1}$.

We study these two cases separately in the following propositions.

Proposition 4.4. For case $(a), \varphi_{14}$ is birational.

Proof. We know that $M_{5}=g_{5}^{*}\left(H_{5}^{\prime}\right)$ for a very ample divisor $H_{5}^{\prime}$ on $W_{5}^{\prime}$ with $H_{5}^{\prime 2}=2$ and $g_{5}: X^{\prime} \longrightarrow W_{5}^{\prime}$ is the birational morphism. Because $W_{5}^{\prime}$ is already normal (which is a cone), by taking further modification to $X^{\prime}$, we can assume that $g_{5}$ factors through the minimal resolution $\mathbb{F}_{2}$ of $W_{5}^{\prime}$. So we have the map $g_{5}: X^{\prime} \stackrel{h_{5}}{\longrightarrow} \mathbb{F}_{2} \stackrel{\nu}{\longrightarrow} W_{5}^{\prime}$ where $h_{5}$ is a fibration and $\nu$ is the minimal resolution. Set $\bar{H}_{5}=\nu^{*}\left(H_{5}^{\prime}\right)$. Then $\bar{H}^{2}=\left(H_{5}^{\prime}\right)^{2}=2$. Noting that $\bar{H}_{5}$ is nef and big on $\mathbb{F}_{2}$, we can write

$$
\bar{H} \sim \mu G_{0}+n T
$$

where $G_{0}$ is the unique section with $G_{0}^{2}=-2, \mu$ and $n$ are integers and $T$ is the general fiber of the ruling on $\mathbb{F}_{e}$. The property of $\bar{H}_{5}$ being nef and big implies $\mu>0$ and $n \geq 2 \mu \geq 2$. Now let $\theta_{2}: \mathbb{F}_{2} \longrightarrow \mathbb{P}^{1}$ be the ruling, whose fibers are all smooth rational curves. Set $f_{0}:=\theta_{2} \circ h_{5}: X^{\prime} \longrightarrow \mathbb{P}^{1}$, which is 
a fibration with connected fibers. Denote by $\tilde{F}$ a general fiber of $f_{0}$ and by $\tilde{\sigma}: \tilde{F} \longrightarrow \tilde{F}_{0}$ the contraction onto the minimal model. Clearly $p_{g}(\tilde{F})>0$. We have

$$
M_{5} \sim g_{5}^{*}\left(H_{5}^{\prime}\right)=h_{5}^{*}(\bar{H}) \geq 2 \tilde{F} .
$$

Set $N_{5}=2 \tilde{F}$. Replace $\left|M_{5}\right|$ by the sub-linear system $\left|N_{5}\right|$ in Theorem 3.1. We can also study the birationality of $\varphi_{m}$ as in Remarked 3.2.

Note that the fibration $f_{0}: X^{\prime} \longrightarrow \mathbb{P}^{1}$ is the induced fibration by $\Phi_{|2 \tilde{F}|}$. We can repeat a similar argument to that in Subcase 3.2 above. We can verify those conditions in Theorem 3.1.

Because $14 K_{X^{\prime}} \geq 2 \tilde{F}, 3.1(\mathrm{i})$ is satisfied. On the other hand, we have $\mathcal{O}(2) \hookrightarrow f_{0 *} \omega_{X^{\prime}}^{5}$ which implies the inclusion

$$
f_{0 *} \omega_{X^{\prime} / \mathbb{P}^{1}}^{2} \hookrightarrow f_{0 *} \omega_{X^{\prime}}^{12}
$$

Similarly Viehweg's semi-positivity and the base point freeness of $\left|2 \sigma^{*}\left(K_{\tilde{F}_{0}}\right)\right|$ say that $\mid 12 K_{X^{\prime}} \|_{\tilde{F}}$ can separate different generic irreducible elements of $\left|2 \sigma^{*}\left(K_{\tilde{F}_{0}}\right)\right|$. So can $\mid 14 K_{X^{\prime}} \|_{\tilde{F}}$. So 3.1(ii) is satisfied.

Now we take $m_{0}^{\prime}=5$ to run Theorem 3.1 with $\left|M_{5}\right|$ replaced by $\left|N_{5}\right|$. One has $p=2$.

If $\tilde{F}$ is of type (III), we take $\tilde{G}:=2 \tilde{\sigma}^{*}\left(K_{\tilde{F}_{0}}\right)$. Take $m=14$. The proof of Lemma 3.3 says that one can take a $\beta \mapsto \frac{1}{7}$ such that $\left.\pi^{*}\left(K_{X}\right)\right|_{\tilde{F}}-2 \beta \tilde{\sigma}^{*}\left(K_{\tilde{F}_{0}}\right)$ is numerically equivalent to an effective $\mathbb{Q}$-divisor. So $\alpha=\left(m-1-m_{0}^{\prime} / p-\right.$ $1 / \beta) \tilde{\xi}>0$. Noting that a generic irreducible element of $|\tilde{G}|$ is nonhyperelliptic and even, $\varphi_{14}$ is birational by Theorem 3.1.

If $\tilde{F}$ is of type (II), we set $\tilde{G}:=\tilde{\sigma}^{*}\left(K_{\tilde{F}_{0}}\right)$. Then $\tilde{C}$, as a generic irreducible element of $|\tilde{G}|$, is smooth and of genus 3 . We have already showed above that 3.1(ii) is satisfied for $m=14$. By Lemma 3.3 we can take a $\beta \mapsto \frac{2}{7}$ such that $\left.\pi^{*}\left(K_{X}\right)\right|_{\tilde{F}}-\beta \tilde{C}$ is numerically equivalent to an effective $\mathbb{Q}$-divisor. So $\tilde{\xi}=\left.\pi^{*}\left(K_{X}\right)\right|_{\tilde{F}} \cdot \tilde{C} \geq \beta \tilde{C}^{2} \mapsto \frac{4}{7}$. Taking the limit one has $\tilde{\xi} \geq \frac{4}{7}$. We have $p=2$. Take $m=14$. Then $\alpha=\left(m-1-\left(m_{0}^{\prime} / p\right)-(1 / \beta)\right) \tilde{\xi} \geq 4$. Theorem 3.1 says that $\varphi_{14}$ is birational.

If $\tilde{F}$ is of type (I), we take $\tilde{G}$ to be the movable part of $\left|\tilde{\sigma}^{*}\left(K_{\tilde{F}_{0}}\right)\right|$. Then $\tilde{C}$, as a generic irreducible element of $|\tilde{G}|$, is smooth and of genus 2 . We have already showed above that 3.1(ii) is satisfied for $m=14$. By Lemma 3.3, we can take $\beta=\frac{2}{7}$ such that $\left.\pi^{*}\left(K_{X}\right)\right|_{\tilde{F}}-\beta \tilde{\sigma}^{*}\left(K_{\tilde{F}_{0}}\right)$ is numerically equivalent to an effective $\mathbb{Q}$-divisor. So $\tilde{\xi}=\left.\pi^{*}\left(K_{X}\right)\right|_{\tilde{F}} \cdot C \geq \beta=\frac{2}{7}$. Take $m=12$. Then $\alpha=\left(m-1-m_{0}^{\prime} / p-1 / \beta\right) \tilde{\xi}>1$. Theorem 3.1 gives $\tilde{\xi} \geq \frac{1}{3}$. Take $m=14$. Then $\alpha=\left(m-1-m_{0}^{\prime} / p-1 / \beta\right) \tilde{\xi} \geq \frac{7}{3}>2$. Theorem 3.1 says that $\varphi_{14}$ is birational. 
Proposition 4.5. For case (b), $\varphi_{14}$ is birational.

Proof. Recall that we have two fibrations $f: X^{\prime} \longrightarrow B$ and $f_{5}: X^{\prime} \longrightarrow W_{5}^{\prime}=$ $\mathbb{P}^{1} \times \mathbb{P}^{1}$. For a general canonical curve $C$ in the a general fiber $F$ of $f$, we can study $\operatorname{dim} \varphi_{5}(C)$. If $\operatorname{dim} \varphi_{5}(C)=1$, then $\Phi_{\left|M_{5}\right|}$ maps $C$ onto a curve. Clearly $5 \pi^{*}\left(K_{X}\right) \cdot C \geq M_{5} \cdot C \geq 2$. So $\xi \geq \frac{2}{5}>\frac{1}{3}$ and $\varphi_{14}$ is birational according to the statement $(* *)$. From now on, we assume that $\operatorname{dim} \varphi_{5}(C)=0$ for a general $C$. We may take further blowing ups to $X^{\prime}$ so that a general $C$ is simply a generic fiber of $f_{5}$.

Because the only very ample divisor $H_{5}^{\prime}$ on $W_{5}^{\prime}$ with $H_{5}^{\prime 2}=2$ is $L_{1}+L_{2}=$ $q_{1}^{*}$ (point) $+q_{2}^{*}$ (point) where $q_{1}, q_{2}$ are projection maps from $\mathbb{P}^{1} \times \mathbb{P}^{1}$ to $\mathbb{P}^{1}$. Set $\tilde{f}_{i}:=q_{i} \circ f_{5}: X^{\prime} \longrightarrow \mathbb{P}^{1}, i=1,2$. Then $\tilde{f}_{1}$ and $\tilde{f}_{2}$ are two fibrations onto $\mathbb{P}^{1}$. Let $F_{1}$ and $F_{2}$ are respectively general fibers of $\tilde{f}_{1}$ and $\tilde{f}_{2}$. Then $F_{1} \cap F_{2}$ is simply a general fiber $C$ of $f_{5}$. We will prove alternatively that $\varphi_{14}$ is birational.

Consider the sub-liner system $\left|K_{X^{\prime}}+\left\ulcorner 8 \pi^{*}\left(K_{X}\right)\right\urcorner+F_{1}+F_{2}\right| \subset\left|14 K_{X^{\prime}}\right|$ which clearly separates different fibers of $\tilde{f}_{1}$. Take a general $F_{1}$ as a fiber of $\tilde{f}_{1}$. Because $8 \pi^{*}\left(K_{X}\right)+F_{2}$ is nef and big, the Kawamata-Viehweg vanishing theorem gives a surjective map

$$
H^{0}\left(K_{X^{\prime}}+\left\ulcorner 8 \pi^{*}\left(K_{X}\right)\right\urcorner+F_{2}+F_{1}\right) \longrightarrow H^{0}\left(F_{1}, K_{F_{1}}+\left.\left\ulcorner 8 \pi^{*}\left(K_{X}\right)\right\urcorner\right|_{F_{1}}+C\right) .
$$

We hope to prove that $\left|K_{F_{1}}+\left\ulcorner 8 \pi^{*}\left(K_{X}\right)\right\urcorner\right|_{F_{1}}+C \mid$ gives a birational map for a general $F_{1}$. Note that $|C|$ is a rational pencil on the surface $\left|F_{1}\right|$. Clearly $\left|K_{F_{1}}+\left\ulcorner 8 \pi^{*}\left(K_{X}\right)\right\urcorner\right|_{F_{1}}+C \mid$ separate different $C$ simply because $p_{g}\left(F_{1}\right)>0$ and $\left.\left\ulcorner 8 \pi^{*}\left(K_{X}\right)\right\urcorner\right|_{F_{1}} \geq 0$. Take a general curve $C$ in the family $\left|F_{2}\right| F_{1} \mid$. The vanishing theorem again gives the surjective map

$$
H^{0}\left(F_{1}, K_{F_{1}}+\left\ulcorner\left. 8 \pi^{*}\left(K_{X}\right)\right|_{F_{1}}\right\urcorner+C\right) \longrightarrow H^{0}\left(C, K_{C}+D\right)
$$

where $D:=\left.\left(\left\ulcorner\left. 8 \pi^{*}\left(K_{X}\right)\right|_{F_{1}}\right\urcorner\right)\right|_{C}$ is a divisor of

$$
\operatorname{deg}(D) \geq\left(\left.8 \pi^{*}\left(K_{X}\right)\right|_{F_{1}}\right) \cdot C=8 \pi^{*}\left(K_{X}\right) \cdot C=8 \xi \geq \frac{8}{3}>2
$$

recalling the inequality (4.2) and that $C$ is also a canonical curve in the surface $F$. So $\Phi_{\left|K_{C}+D\right|}$ is an embedding and the birationality principle says that $\varphi_{14}$ is birational. We have completely showed Theorem 4.2.

Example 4.6. Example 1.2 shows that Theorem 4.2 is optimal for $m_{0}=3$. It is also optimal for $m_{0}=2$. In fact, Fletcher has an example 
([10, No. 18, p. 151]): the canonical hypersurface $X_{22} \subset \mathbb{P}(1,2,3,4,11)$. It is clear $m_{0}=2$ and that $\varphi_{11}$ is birational, but $\varphi_{10}$ is not birational.

Recently we were informed of a new construction of a 3 -fold $Y$ by E. Stagnaro [27]. $Y$ also has $p_{g}=1, P_{2}=2$ and $\varphi_{10}$ is not birational.

By a similar method, we are able to prove Corollary 1.3. To avoid unnecessary redundancy we omit some details.

\subsection{A sketch proof of Corollary 1.3}

First one has $P_{2}(X) \geq 4$ by a similar application of Reid's formula (see the proof of Lemma 4.1). So one may take $m_{0}=2$. Set $d_{2}:=\operatorname{dim} \varphi_{2}(X)$.

If $d_{2} \geq 2$, it is clear that 3.1 (i), (ii) are satisfied for $m \geq 8$. Now the situation $d_{2}=3$ follows directly from the argument in Case 1 of Theorem 4.2. In fact, one get the birationality of $\varphi_{7}$. In the situation $d_{2}=2$, one may also follow the argument in Case 2 of Theorem 4.2. The only difference is that we can take $p=2$ here. So it can be verified that $\varphi_{8}$ is birational.

If $d_{2}=1$, one has an induced fibration $f: X^{\prime} \longrightarrow B$. When $g(B)>0$, the argument in Subcase 3.1 of Theorem 4.2 shows that $\varphi_{m}$ is birational for all $m \geq 7$. We assume $g(B)=0$ from now on. Let $F$ be a general fiber of $f$. If $p_{g}(F)=0$, then $q(X)=q(F)=0$ and the assumption $\chi\left(\omega_{X}\right)>0$ implies $p_{g}(X) \geq 2$. So $\varphi_{m}$ is birational for all $m \geq 8$ by the main theorem in [8]. We are reduced to study the situation $p_{g}(F)>0$. Now we can follow the argument of Subcase 3.2 in Theorem 4.2. The typical property here is the inclusion

$$
\mathcal{O}(3) \hookrightarrow f_{*} \omega_{X^{\prime}}^{2}
$$

We can still choose $G \leq 2 \sigma^{*}\left(K_{F_{0}}\right)$ on $F$. We can verify that Theorem 3.1 (i), (ii) are satisfied for all $m \geq 6$. Also we can choose much better $p(p \geq 2)$ and $\beta$, respectively, than those in Subcase 3.2 in Theorem 4.2. We can verify with less difficulties, case by case (for type (I), (II), (III)) by applying Theorem 3.1, that $\varphi_{m}$ is birational for all $m \geq 8$.

In a word, Corollary 1.3 is true.

\subsection{Final remarks}

Clearly, for the case $p_{g}=0$, Theorem 3.1 will generate some new results which improves a corollary of J. Kollár [21] and the first author on the birationality of $\varphi_{m}$, where $m$ is certain function in terms of $m_{0}$. We do not include our result here because we are not sure whether that would be optimal. 


\section{Acknowledgments}

This paper was supported by both the Program for New Century Excellent Talents in University (\#NCET-05-0358) and the National Outstanding Young Scientist Foundation (\#10625103).

\section{References}

[1] W. Barth, C. Peter and A. Van de Ven, Compact complex surface, Springer-Verlag, Berlin, 1984.

[2] E. Bombieri, Canonical models of surfaces of general type, Inst. Hautes tudes Sci. Publ. Math. 42 (1973), 171-219.

[3] F. Catanese and C. Ciliberto, Surfaces with $p_{g}=q=1$. Problems in the theory of surfaces and their classification (Cortona, 1988), Symposia Mathematica, XXXII, Academic Press, London, 1991, 49-79.

[4] J.A. Chen, M. Chen and D.-Q. Zhang, The 5-canonical system on threefolds of general type, J. Reine. Angew Math. 603 (2007), 165-181.

[5] J.A. Chen and M. Chen, Explicit birational geometry of threefolds of general type, Preprint, 2007 arXiv:0706.2987.

[6] J.A. Chen and C. Hacon, Linear series of irregular varieties, Algebraic Geometry in East Asia (Kyoto, 2001), World Sci. Publ., River Edge, NJ, 2002, 143-153.

[7] J. A. Chen and C. Hacon, Pluricanonical systems on irregular 3-folds of general type, Math. Z. 255 (2) (2007), 343-355.

[8] M. Chen, Canonical stability of 3-folds of general type with $p_{g} \geq 3$, Internat. J. Math. 14 (2003), 515-528.

[9] M. Chen, Canonical stability in terms of singularity index for algebraic threefolds, Math. Proc. Camb. Philos. Soc. 131 (2001), 241-264.

[10] A. Corti and M. Reid, Explicit birational geometry of 3-folds, London Mathematical Society, Lecture Note Series, 281, Cambridge University Press, Cambridge, 2000.

[11] P. Francia, On the base points of the bicanonical system, Problems in the theory of surfaces and their classification (Cortona, 1988), 
Symposia Mathematica, XXXII, Academic Press, London, 1991, 141-150.

[12] M. Green and R. Lazarsfeld, Deformation theory, generic vanishing theorems and some conjectures of Enriques, Catanese and Beauville, Invent. Math. 90 (1987), 389-407.

[13] C.D. Hacon and J. McKernan, Boundedness of pluricanonical maps of varieties of general type, Invent. Math. 166 (1) (2006), 1-25.

[14] C.D. Hacon, J. McKernan, Shokurov's rational connectedness conjecture, Duke Math. J. 138 (1) (2007), 119-136.

[15] A.R. Iano-Fletcher, Inverting Reid's exact plurigenera formula. Math. Ann. 284 (41) (1989), 617-629.

[16] J. Jost and K. Zuo, Vanishing theorems for $L^{2}$-cohomology on infinite coverings of compact Kahler manifolds and applications in algebraic geometry, Comm. Anal. Geom. 8 (1) (2000), 1-30.

[17] Y. Kawamata, A generalization of Kodaira-Ramanujam's vanishing theorem, Math. Ann. 261(1982), 43-46.

[18] Y. Kawamata, Characterization of abelian varieties, Compositio Math. 43 (2) (1981), 253-276.

[19] Y. Kawamata, K. Matsuda and K. Matsuki, Introduction to the minimal model problem, Adv. Stud. Pure Math. 10 (1987), 283-360.

[20] J. Kollár, Shafarevich maps and automorphic forms, Princeton University Press, Princeton, New Jersey, 1995.

[21] J. Kollár, Higher direct images of dualizing sheaves I, Ann. Math. 123 (1986), 11-42; II, ibid. 124 (1986), 171-202.

[22] J. Kollár and S. Mori, Birational geometry of algebraic varieties, University Press, Cambridge, 1988.

[23] M. Reid, Young person's guide to canonical singularities, Proc. Sympos. pure Math. 46 (1987), 345-414.

[24] M. Reid, Chapters on algebraic surfaces, Complex algebraic geometry. (Park City, UT, 1993), IAS/Park City Mathematical Series, 3, American Mathematical Society, Providence, RI, 1997, 3-159.

[25] I. Reider, Vector bundles of rank 2 and linear systems on algebraic surfaces, Ann. Math. 127 (1988), 309-316. 
[26] V.V. Shokurov, On rational connectedness, Math. Notes 68 (2000), 652-660.

[27] E. Stagnaro, Threefolds with Kodaira dimension 0 or 3, Preprint, 2006.

[28] S. Takayama, Pluricanonical systems on algebraic varieties of general type, Invent. Math. 165 (3) (2006), 551-587.

[29] S.G. Tankeev, On n-dimensional canonically polarized varieties and varieties of fundamental type, Izv. A. N. SSSR, Ser. Math. 35 (1971), 31-44.

[30] G. T. Todorov, Pluricanonical maps of threefolds of general type, Preprint, 2005 arXiv:math.AG/0512346

[31] H. Tsuji, Pluricanonical systems of projective 3-folds of general type, Preprint, 2002 arXiv:math.AG/0204096

[32] E. Viehweg, Vanishing theorems, J. Reine Angew. Math. 335 (1982), $1-8$.

[33] E. Viehweg, Weak positivity and the additivity of the Kodaira dimension for certain fibre spaces, Proc. Algebraic varieties and analytic varieties, (Tokyo 1981). Advanced Studies in Mathematics, 1, Kinokunya-NorthHolland Publ., 1983, 329-353

Institute OF MATHEMATICS

SChool of Mathematical Sciences

FUDAN UNIVERSITY

SHANGHAI 200433

CHINA

E-mail address: mchen@fudan.edu.cn

Faculty of Mathematics and Computer Science

Johannes Gutenberg Universitaet Mainz

STAUDINGERWEG 9

D-55128 MAINZ

GERMANY

E-mail address: kzuo@mathematik.uni-mainz.de 